\title{
2.2 A phase II trial with canakinumab, a new IL- I beta blocking monoclonal antibody (ACZ885), to evaluate preliminary dosing, safety and efficacy profile in children with systemic Juvenile Idiopathic arthritis (sJIA)
}

N Ruperto*1, P Quartier ${ }^{2}$, N Wulffraat ${ }^{3}$, P Woo ${ }^{4}$, A Loy ${ }^{1}$, R Mouy², B BaderMeunier ${ }^{2}$, B Prakken ${ }^{3}$, E Noseda ${ }^{5}$, C Rordorf ${ }^{5}$ and Martini for the Paediatric Rheumatology International Trials Organisation (PRINTO) $\mathrm{A}^{1}$

Address: ${ }^{1}$ IRCCS G. Gaslini, Pediatria II - PRINTO, Largo Gaslini, Genova, Italy, ${ }^{2}$ Hospital Necker Enfants Malades, Unit'e d'Immunologie, Hematologie et Rhumatologie Pediatrique, Paris, France, ${ }^{3}$ Dept pediatric immunology, University Medical Center Utrecht, Utrecht, Netherlands, ${ }^{4}$ Centre of Paediatric and Adolescent Rheumatology, The Windeyer Institute, London, UK and ${ }^{5}$ Novartis Pharma AG, Basel, Switzerland

* Corresponding author

from 15th Paediatric Rheumatology European Society (PreS) Congress

London, UK. 14-17 September 2008

Published: 15 September 2008

Pediatric Rheumatology 2008, 6(Suppl I):S2 doi:I0.I I86/I546-0096-6-SI-S2

This abstract is available from: http://www.ped-rheum.com/content/6/SI/S2

(c) 2008 Ruperto et al; licensee BioMed Central Ltd.

\section{Objectives}

Phase II trial to evaluate dosing and interval range, preliminary efficacy, safety, immunogenicity, and pharmacokinetics of subcutaneous (s.c.) canakinumab in patients with active sJIA.

\section{Methods}

19 children 4-19 years old, with fever, at least 2 active joints, CRP > $50 \mathrm{mg} / \mathrm{L}$ and steroids $\leq 0.4 \mathrm{mg} / \mathrm{kg}$, were enrolled in an open label, staggered dose escalation study. Patients received a single sc injection of canakinumab in the dose range $0.5-9 \mathrm{mg} / \mathrm{kg}$, followed by an observation period and re-dosing upon relapse. Dose escalation was based on safety and efficacy review of each cohort. Response was measured according to modified ACR pediatric criteria, (at least 3/6 variables improved by $\geq 30 \%$ with no more than one variable worsening by $>30 \%$ and no fever). Relapse was defined as reappearance of fever and CRP > $30 \mathrm{mg} / \mathrm{L}$, and/or ACR pediatric flare criteria.

\section{Results}

11/19 patients responded to canakinumab. At Day 15 post first dose all the 11 responders achieved at least an ACR pediatric 50. In 4 cases inactive disease status was reached (no joints with active arthritis, no fever, normal CRP and no disease activity according to physician's assessment). Time to relapse after first dose ranged from 23 days to $>200$ days. The injections were well tolerated and no immunogenicity developed. One serious adverse event (gastritis with ulcer bleeding) was reported.

\section{Conclusion}

In this dose-escalation trial canakinumab was efficacious and provided improvement in sign and symptoms of sJIA with an acceptable safety profile. 